Meta

Journal des traducteurs

Translators' Journal

\title{
Traitement terminologique : de la peau au cuir
}

\section{Mylène Cartier, Marie-Louise Hannan, Nadia Moulay et Jean Ntakirutimana}

Volume 40, numéro 1, mars 1995

URI : https://id.erudit.org/iderudit/002208ar

DOI : https://doi.org/10.7202/002208ar

Aller au sommaire du numéro

Éditeur(s)

Les Presses de l'Université de Montréal

ISSN

0026-0452 (imprimé)

1492-1421 (numérique)

Découvrir la revue

Citer cet article

Cartier, M., Hannan, M.-L., Moulay, N. \& Ntakirutimana, J. (1995). Traitement

terminologique : de la peau au cuir. Meta, 40(1), 99-129.

https://doi.org/10.7202/002208ar

\section{Résumé de l'article}

Les auteurs, un groupe d'étudiants, proposent une méthodologie terminologique qu'ils appliquent au domaine du cuir. La recherche bibliographique, l'élaboration de l'arbre du domaine, la mise au point de la fiche informatisée et des questions théoriques sont traitées dans le cadre du cours "Terminologie théorique et pratique 2" dispensé par le professeur André Clas. 
électricité : les verbes exprimant la relation de liaison. "Pourquoi la porte ne tourne-t-elle pas sur ses gonds et l'étoile n'oscille-t-elle pas sur son axe?» La réponse est dans le verbe.

Massiva N. ZaFiO

Universite du Québec a Trois-Rivieres. Trois-Rivieres, Canada

Notes

1. Cette définition de la collocution, proposée par Ducrot et Todorov, est irès eloigné de celle qu'en donnent Ménard (1989) et Francine Bergevin (1991). Ces demiers considerent la collocation comme un phénomène régi par des contraintes semantiques, ce qui justifie sa prise en comple dans la mesure des relations lexicosémantiques. pour l'un, et dans l'étude de la cohesion, pour l'autre.

\section{REFERENCES}

BOONS. Jean-Paul (1985) : „Preliminaires à la classification des verbes locatifs : les compléments de lieu, leurs valeurs aspectuellesm, Linguistica Investigationes, IX, $n^{\circ} 2$, Amsterdam, John Benjamins.

BOONS, Jean-Paul (1987) : «a notion sémantique de déplacement dans une classification syntaxique des verbes Iocatifsw (photocopie).

BOL CHARD, Denis (à paraitre): The Semantics of Syntax.

CRESSOT, Marcel $(1966)$ : Le style et ses techniques, Paris, Hachette.

DARBELNET, Jean (1988) : «L'apport de la stylistique comparte à l'enseignement de la traduction*, Meta, 33-2, pp. 13.3-141.

DUBOIS ef al. (1973) : Dictionnaire de linguistique. Paris, Larousse.

LAMIROY, Beatrice (1983): Les verbes de mouvement en fransais ef en espagnol, Amsterdam et Philadelphie, Presses de I'Universite de Louvain.

LETHUILLIER, Jacques ( 1991 ) : „Combinatoire, terminologies et textes*, Meta, 36-1, pp. 92-100.

LYONS, John (1977): Semantics, Cambridge, Cambridge University Press.

MAINGENEAU, Dominique (|(9)|) : L'analvse du discours. Introduction aux lectures de l'archive, Paris, Hachette.

MÉNARD. Nathan (1989) : «Mesure des relations lexico-semantiques dans des textes scientifiques: problemes méthodologiques». Meta. 34-3, pp. 468-478.

VANDELOISE, Claude (1986) : L'espace en fransais - Semantique des prépositions spatiales, Paris, Editions du Seuil.

\section{TRAITEMENT TERMINOLOGIQUE : DE LA PEAU AU CUIR}

\section{Résumé}

Les auseurs, un groupe d'érudiants, proposent une méthodologie terminologique qu'ils appliquent au domaine du cuir. La recherche bibliographique. l' elaboration de l'arbre du domaine, la mise au point de la fiche informatisée et des questions théoriques sont traitées dans le cadre du cours "Terminologie théorique et pratique 2 ¿dispensé par le professeur André Clas.

\section{POURQUOI LE CUIR ?}

Notre choix s'est porté sur le cuir pour diverses raisons. La première est une raison d'objectif du travail terminologique. En effet, nous avions pour but d'appliquer une méthode de recherche et d'en vérifier l'efficacité, ce qui nous donnait toute latitude pour choisir le domaine d'études. Il fallait toutefois que ce demier soit suffisamment technique et que la recherche puisse s'effectuer dans des délais raisonnables et être intéressante pour les terminologues. 
La démarche adoptée commence par la familiarisation avec le sujet: elle se poursuit avec l'établissement d'une bibliographie structurée à l'aide de critères de fiabilité, et se termine par l'élaboration de l'arbre des domaines et par la mise au point d'une fiche informatisée.

\section{EXPLORATION DU SUJET}

Avant d'entreprendre une recherche, il faut en explorer le sujet. Une première consultation d'ouvrages généraux sur le cuir a été effectuée.

Le Que sais-je? intitulé Le cuir nous donne une description précise des différentes étapes de la transformation des peaux en cuir fini, en plus d'expliquer les processus, les techniques. et les outils utilisés pour chacune de ces étapes. Cependant, cet ouvrage datant de 1947. nous l'avons abordé avec certaines réserves.

Dans l'Encyclopecdia Universalis, nous avons obtenu le même genre de renseignements mais les descriptions des étapes de la transformation sont plus concises et les sources sont plus récentes. De plus, il y est question des notions de qualité des cuirs et d'examens des cuirs.

Dans la famille Larousse (Grand Larousse de la Langue fransaise. Grand Larousse Encyclopédique, Larousse en trois volumes), nous avons trouvé peu de détails sur les aspects techniques. En revanche, les articles sont riches du point de vue terminologique; en outre. ils soulèvent certains problèmes de ce même ordre. comme par exemple la distinction cuir-peau.

Nous avons aussi consulté quelques ouvrages en anglais. L'Encyclopaedia Britannica donne très peu de renseignements sur le sujet. En fait, elle ne fournit que quelques termes.

L'Encyclopédie du Canada donne une description des aspects sociaux et historiques du travail du cuir. Nous y trouvons aussi de nombreux termes de métiers et des faits intéressants sur l'histoire des tanneries.

Nous avons également consulté le Colliers' Concise Encyctopedia et The World Book. Ces deux ouvrages expliquent les étapes de la transformation. et donnent la définition d'un grand nombre de termes.

Ces lectures générales nous ont permis de nous familiariser avec les diverses phases de la transformation des peaux en cuir fini. Toutefois, nous devions encore approfondir nos connaissances afin de pouvoir relever la terminologie pertinente. Nous avons donc entrepris la recherche bibliographique.

\section{RECHERCHE BIBI.IOG:RAPHIQUE}

Afin d'établir une bonne bibliographie, nous avons relevé ce qui pouvait concerner le cuir, sans nous soucier de la direction que prendrait notre recherche au moment d'élaborer l'arbre du domaine. Ainsi, nous avons établi une bibliographie de près de 140 titres. Il fallait alors trouver des moyens pour classer la documentation.

Nous avons d'abord décidé de limiter le sujet aux étapes de la transformation des peaux et d'exclure tout ce qui concernait les produits chimiques utilisés dans ces étapes ainsi que les produits finis du cuir. En procédant ainsi, nous avons pu retirer 56 titres à notre bibliographie.

Puis, nous avons divisé notre documentation en deux types de sources: les sources primaires, qui comprennent les normes, les brevets, les actes de colloques, les ouvrages spécialisés et les manuels de formation, et les sources secondaires, qui incluent les lexiques, les glossaires, les dictionnaires et les encyclopédies. Comme elles sont plus fiables, les sources primaires constitueront notre documentation principale dans la recherche des termes, des définitions et des contextes. Ce premier niveau de classement nous a permis 
de sélectionner environ 60 documents dans les sources primaires et 20 dans les sources secondaires.

Néanmoins, nous n'avions pas suffisamment réduit le nombre de documents pour pouvoir effectuer un travail en profondeur. Il fallait donc établir une liste de critères spécifiques afin d'évaluer la documentation et d'éliminer les documents les moins pertinents.

Les critères suivants ont été retenus :

a. Date de publication de l'ouvrage : nous avons privilégié la date car il est important d'être au fait des derniers progrès technologiques du domaine étudié. Nous avons par conséquent choisi les ouvrages les plus récents, surtout pour l'outillage et les procédés chimiques, qui semblent avoir évolué. Toutefois, il ne faut pas restreindre le sens de récent: dans un domaine ancien comme celui du cuir, les ouvrages récents peuvent dater de 1960 car les opérations de transformation ayant peu changé, il n'y a peut-être pas eu un nombre considérable de nouveaux écrits techniques sur le sujet.

b. Notoriété de l'auteur et notoriété des organismes: si un auteur a publié plusieurs ouvrages sur un même sujet, on peut conclure à sa compétence : les ouvrages des organismes spécialisés, dont la crédibilité est établie, constituent aussi de très bonnes références.

c. Réédition et tirage de l'ouvrage: ces données révèlent que l'ouvrage a eu un certain succès et qu'il est de bonne qualité ; il ne faut pas oublier que leur grande diffusion contribue aussi à répandre la terminologie.

d. Présence d'un index et d"une bibliographie: cette donnée indique que le travail est élaboré puisqu'il a exigé une recherche approfondie et un choix judicieux des mots; elle apporte davantage de crédibilité à l'ouvrage.

e. Versions traduites: si l'on dispose de suffisamment de documentation en version originale, on peut éliminer les traductions : sinon il s'agira d'utiliser ces dernières avec prudence.

L'application de ces critères' nous a permis de réduire notre bibliographie à 44 documents.

\section{GLABORATION ET VERIFICATION DE L'ARBRE DU DOMAINE}

\section{Elaboration}

Nous avons abordé la partie théorique du travail en nous interrogeant sur l'utilité de l'arbre du domaine en terminologie. Nous avons conclu qu'il permettait de rationaliser les recherches, de repérer facilement des domaines et des sous-domaines, d'éviter des interférences et de faciliter l'élaboration des définitions. L'arbre doit être le plus simple possible afin d'être exploitable au maximum. Par exemple. afin de ne pas surcharger l'arbre et rendre sa compréhension difficile, nous avons décidé de ne pas inclure certaines sous-étapes. Dans le cas du corroyage, par exemple, nous n'avons pas tenu compte des neuf sous-étapes. Enfin, il faut préciser que nous avons élaboré les arbres les uns à la suite des autres pour montrer le raisonnement qui nous a conduits a l'arbre final.

Dès la première lecture des encyclopédies anglaises et françaises, nous avons relevé deux notions qui apparaissaient fréquemment dans les deux langues : celles de cuir et de peaux et celles de hides et de skins. À partir de cette constatation, nous avons ébauché l'arbre 1: 


\section{ARBRE 1}

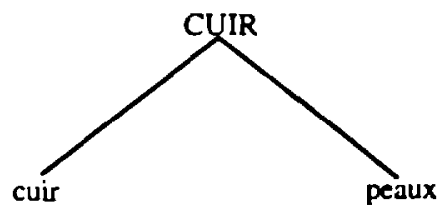

Nous nous demandons alors s`il y a une différence entre "cuir» et "peaux" et entre hides et skins ou si le français est un calque de l'anglais.

En réalité. une ambiguité demeure : le cuir est soit le résultat du tannage de la peau, soit la dépouille du gros bovin après tannage, la peau provenant des petits animaux seulement (GDEL 1982: 2826). Nous décidons de commencer l'arbre 2 avec animal :

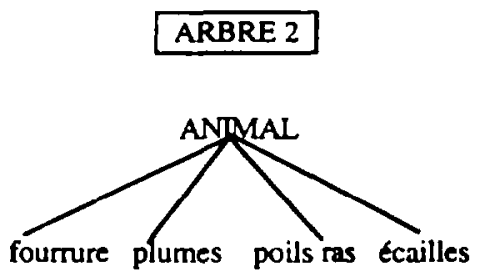

Notre documentation ne traitant que des animaux à partir desquels on fabrique du cuir, nous éliminons les catégories d'animaux à fourrure et à plumes et nous choisissons de prendre le générique «animal» dans le sens «animal dont la dépouille est destinée à la fabrication du cuirm. Nous obtenons ainsi l'arbre 3: 


\section{ARBRE 3}

ANIMAL

(dont la peau donne du cuir)

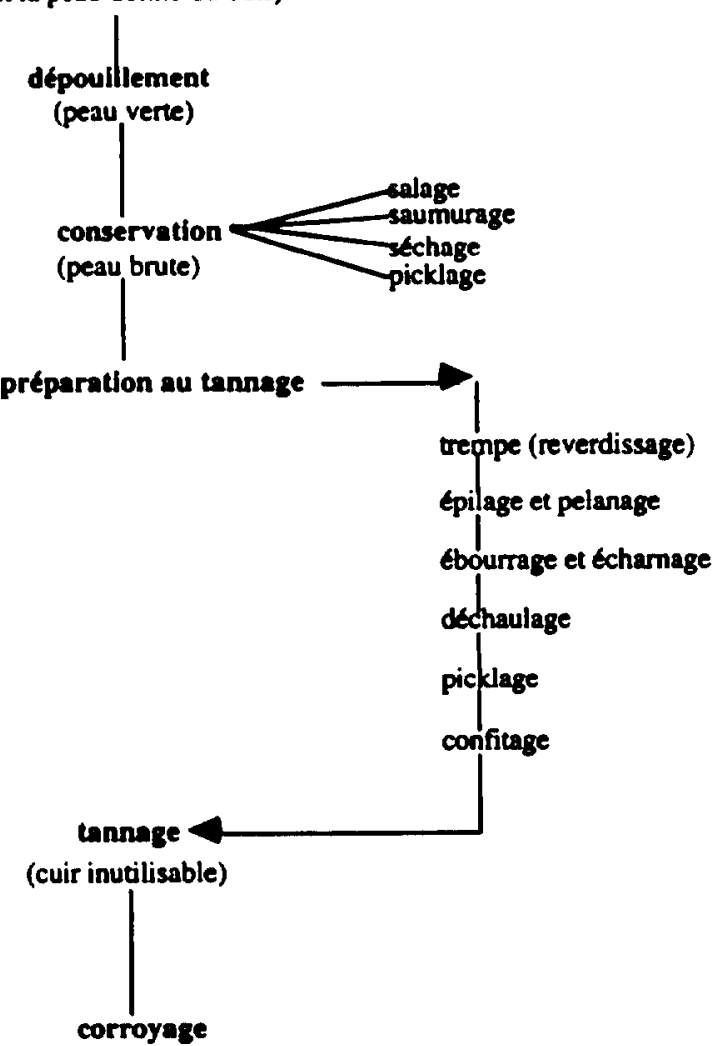

(cuir fini)

Mais procède-t-on de la même façon pour tanner la peau des animaux à écailles et celle des animaux à poils ras? Comme notre documentation ne vise que des animaux à poils ras, nous décidons une fois de plus de modifier notre arbre. Nous voulons aussi ajouter à ce nouvel arbre les types de peaux, leurs défauts et parties, car bon nombre de termes y renvoient. $D$ 'où l'arbre 4 : 


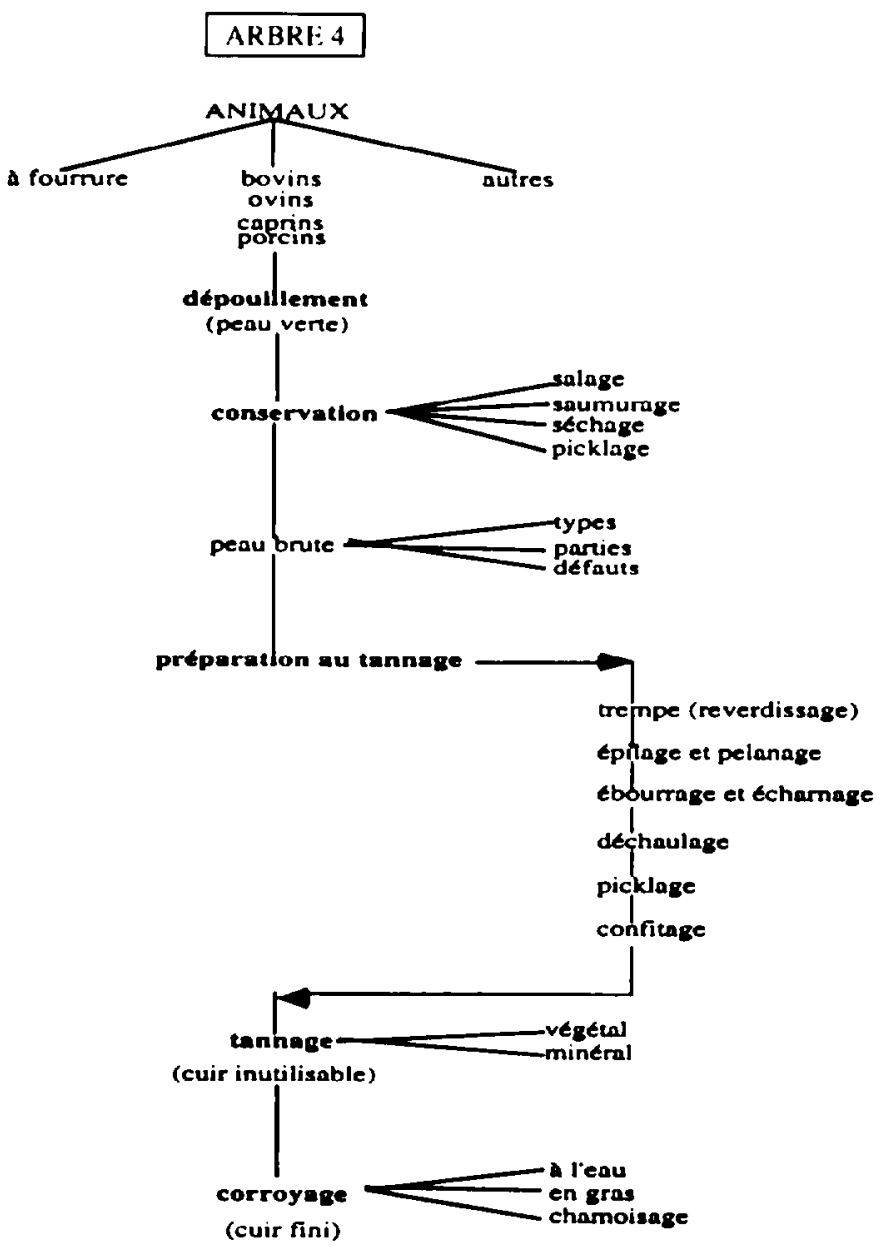

A cette étape-ci, l'élagage se précise:

nous soulignons qu'il s'agit d’animaux abattus, car "animaux» nous paraît trop général. Il aurait pu s'agir d'animaux vivants. et dans ce cas, il aurait fallu ajouter l'étape de l'abattage des animaux. Or, cette dernière relevant de la boucherie, elle ne concerne pas notre champ d'études: la fabrication du cuir, depuis la transformation de la peau jusqu'au cuir fini. Nous nous limitons au résultat de l'abattage : les animaux abattus:

- nous ajoutons les équidés à la liste des animaux dont la dépouille sert à la fabrication du cuir :

- nous précisons que conservation n'est pas un terme, mais bien une étiquette pour les divers modes de conservation de la peau verte. Létiquette sera mise entre guillemets; nous réglons la question de l'emplacement dans l'arbre des caractéristiques de la peau (types, parties et défauts). Leur position dans l'arbre 4 ne suivait pas l'ordre logique des autres parties: 
- nous insérons le type de peau ou de cuir à la suite de l'opération dont il résulte (lorsque le terme existe). Par exemple, conservation (peau brute):

- nous vérifions le sens de «reverdissage». C'est un synonyme de "trempe" (Grand Larousse Encyclopédique).

Une fois ces transformations apportées, nous obtenons l'arbre 5:

\section{ARBRES}

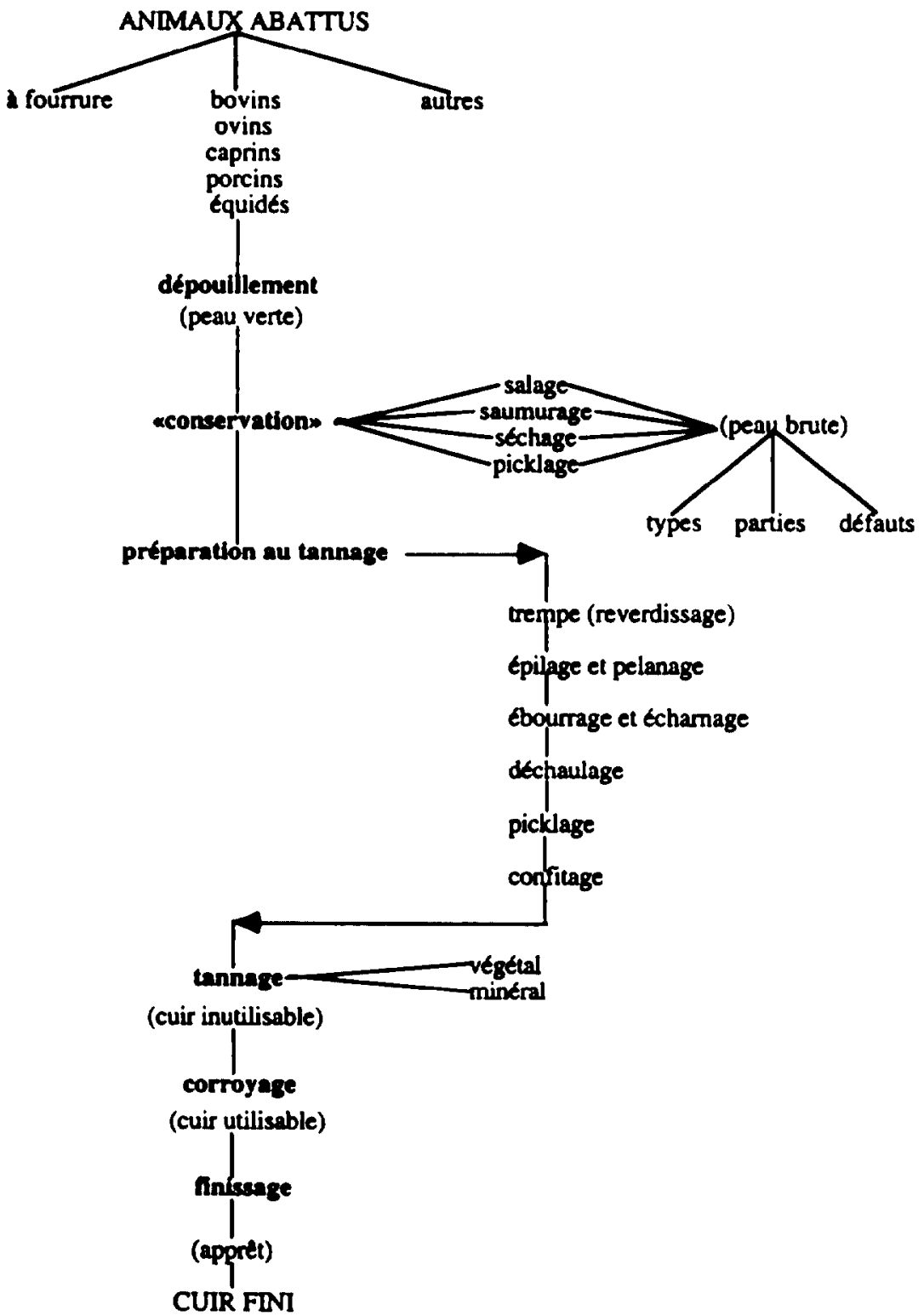


Enfin, nous résolvons les derniers problèmes:

nous signalons que asuivant la nature de cette peau, les entreprises se classent en tanneries, qui traitent les péaux de bovins et d’équidés. et en mégisseries, qui traitent principalement les peaux d'ovins et de caprins" (Universalis 1973: 856). Le traitement est-il différent selon qu iil est fait en tannerie ou en mégisserie ? Comme ce n'est pas le cas, l'arbre ne change pas. Sinon, il aurait fallu ajouter une branche «entreprises de traitement" et deux branches distinctes "traitement en tannerie" et "traitement en mégisserie». En fait, la différence entre «tannerie» et «mégisserie» réside dans le type de peau tannée. On tanne de grosses peaux dans les tanneries et de petites peaux (mouton, chèvre et porc) dans les mégisseries. Par contre, le mode de tannage reste le même:

- nous vérifions le sens de "corroyage" el nous nous apercevons que cette étape doit être plus détaillée que dans l'arbre 5 puisque, par définition. c'est une série d'opérations chimiques et mécaniques par lesquelles le cuir tanné est amené au cuir fini : enfin. nous ajoutons le tannage organique aux deux autres types de tannage.

Nous aboutissons à l'arbre 6 :

\section{ARBRE 6}

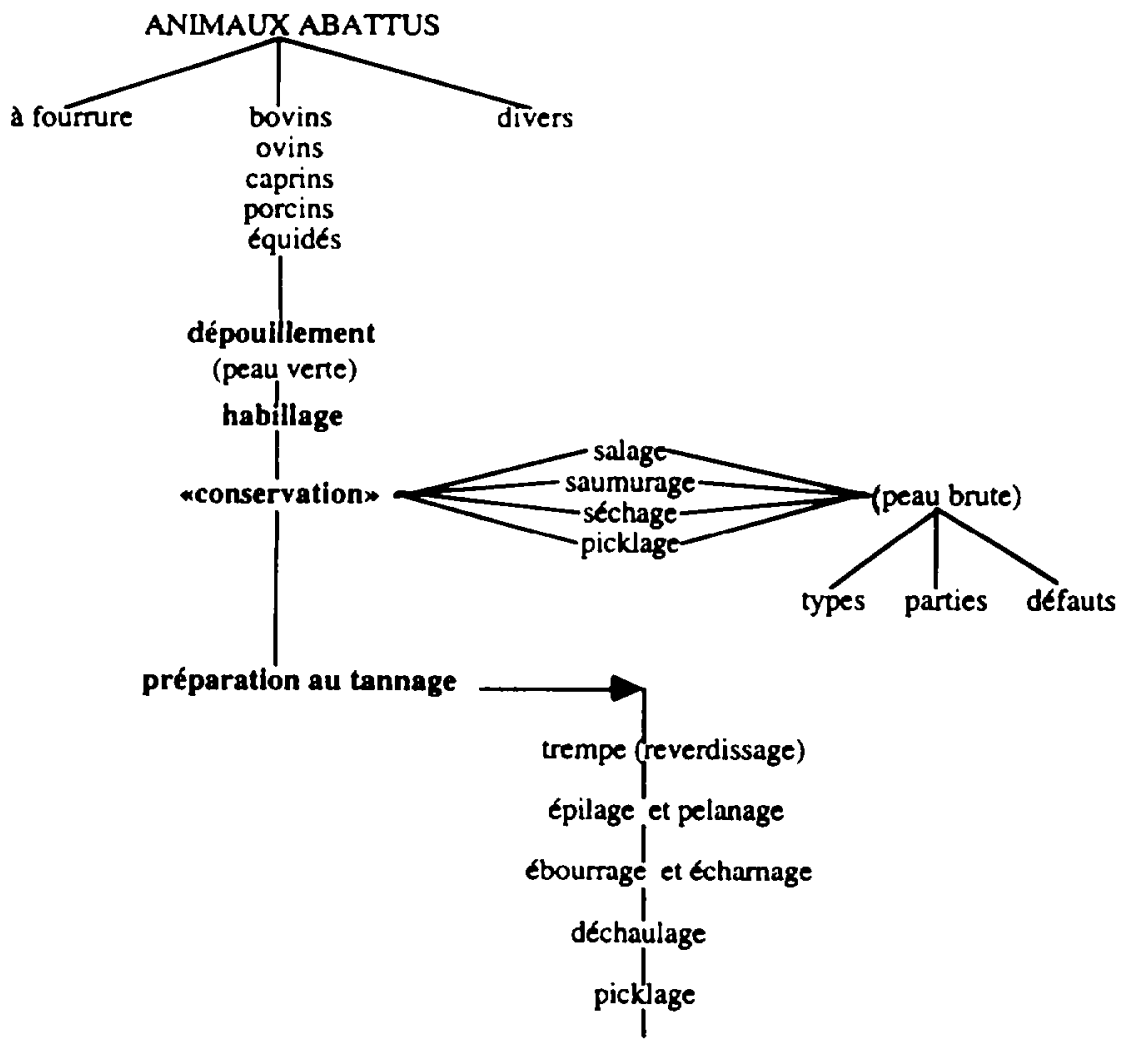




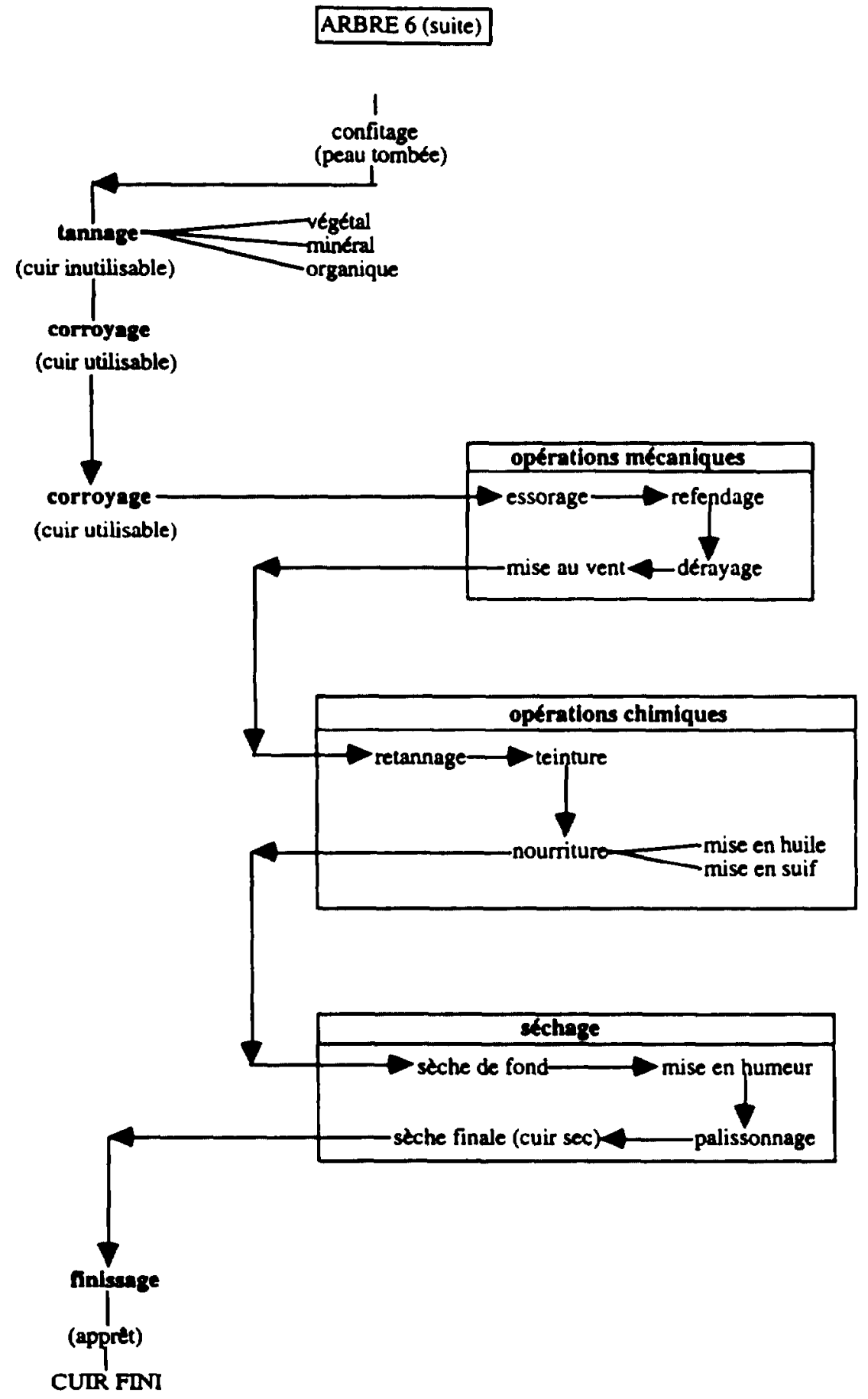




\section{Vérification}

Nous vérifions l'arbre en y insérant le maximum de termes possible. Pour les fins du présent article, nous illustrons les diverses étapes de cette vérification par quelques exemples seulement.

VÉRIFICATION DE L'ARBRE 6
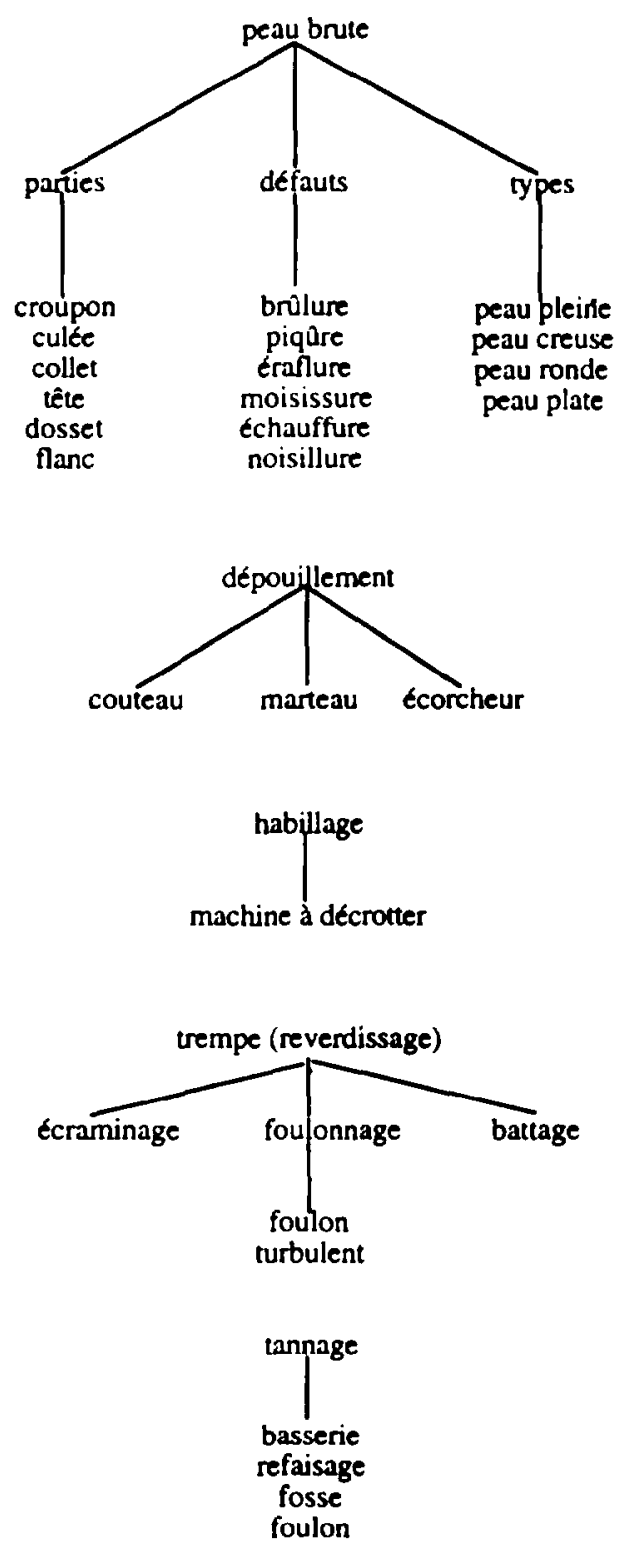


\section{LA FICHE}

\section{Structure de la fiche}

Jusqu'à présent, il n'existe pas de modèle unique de fiche terminologique : les modèles varient selon le domaine, les auteurs, les besoins des utilisateurs, l'importance des banques terminologiques.

Nous avons choisi la fiche bilingue français-anglais mettant en évidence une structure parallèle entre les deux langues.

En outre, il a été décidé que les rubriques suivantes devraient y figurer.

\section{VEDETTE}

Une fiche terminologique doit avoir une vedette, c'est-à-dire le terme principal d'entrée qui régit tous les renseignements terminologiques contenus dans la fiche.

\section{CATÉGORIE GRAMMATICALE}

La catégorie grammaticale est une notion linguistique aux contours flous, car son contenu varie selon la langue décrite.

Dans la pratique lexicographique et terminologique des langues latines, on a pris l'habitude d'utiliser la catégorie grammaticale pour différencier les principales catégories lexicales (entre autres noms, verbes, adjectifs, adverbes), souvent avec ajout de souscatégories, mais l'emploi de la notion de catégorie grammaticale n'est pas le même dans toutes les langues.

Ainsi, nous utiliserons la catégorie grammaticale pour distinguer surtout les noms (n) des verbes $(v)$, afin d'éviter les très fréquentes ambiguîtés dans l'emploi de termes. Nous en profiterons pour préciser le genre masculin (nm) ou féminin (nf) des termes français.

\section{REMARQUES}

Il nous a semblé également nécessaire de réserver, sur chaque fiche, une rubrique remarques, ouverte mais non obligatoire, qui permette d'ajouter, au besoin, les particularités de forme ou d'usage.

\section{SYNONYMES}

Cette section sera remplie pour les vedettes ayant des synonymes ou des variantes orthographiques.

Au départ, nous pensions traiter équitablement tous les termes, en consacrant une fiche complète à chacun d'eux. Cependant, en raison du très grand nombre de synonymes et de variantes orthographiques dans la liste de termes. les fiches risquaient d'être inutilement longues. C'est pourquoi nous avons décidé de consacrer une fiche complète au terme le plus fréquemment utilisé, tandis que les synonymes et les variantes orthographiques font l'objet de fiches partielles, avec renvoi à la fiche principale.

\section{RENVOIS}

Les renvois à la vedette de la fiche principale sont utilisés pour les synonymes ou les variantes orthographiques. Ainsi, il est possible de les relier et surtout de les rattacher à la fiche principale.

\section{DÉFINITION ET CONTEXTE}

La terminologie prône une définition idéale, la plus exacte possible, avec probabilité nulle d'ambiguiité. 
Pour faire face à cette difficulté. la tendance actuelle est de favoriser le contexte plutôt que la définition. Certains se demandent même lequel des deux est le plus important dans une fiche terminologique.

Du reste, la frontière entre définition et contexte n'est pas suffisamment nette. Par exemple, les illustrations de contextes définitoires ${ }^{2}$ fournies par De Bessé (1991 : 112-113) pourraient facilement être prises pour des types de définitions.

Par ailleurs, la distinction semble être un faux débat; en effet, pour Brunette (1992:6), ce sont les informations obtenues à partir des contextes et des définitions qui doivent être prises en compte pour assurer l'appariement non ambigu des notions."

Notre fiche reproduira, dans la mesure des disponibilités des sources, la définition, suivie de la source écrite entre parenthèses, ainsi que le contexte d'utilisation. également suivi de la source entre parenthèses.

\section{COLLOCATIONS}

Il n'est pas toujours facile de trouver les collocations d'un terme : cependant, nous avons décidé de les noter pour chaque terme, et de les mentionner dans la fiche sous la rubrique collocations.

Les collocations nous semblent particulièrement utiles dans une fiche terminologique: en effet, il ne suffit pas de connaitre la signification d'un terme; il faut aussi pouvoir l'utiliser correctement, en particulier dans un discours spécialisé visant une précision optimale.

\section{DATE}

La date est importante dans une fiche puisqu elle indique le moment de la rédaction.

\section{INITIALES DU RÉDACTEUR}

De la même manière qu'il est important de pouvoir dater une fiche, celui qui la consulte voudra également en identifier le rédacteur.

Cependant, compte tenu des dimensions limitées de la fiche, la pratique terminologique a opté pour une identification codée des auteurs.

Dans le cadre du présent travail, la codification du rédacteur de la fiche est constituée des initiales des prénom et nom. d'où la rubrique initiales.

\section{DOMAINE ET SOUS-DOMAINE}

Tous les termes appartiennent au domaine du cuir, objet de notre étude.

Étant donné la complexité du sujet traité (voir l'arbre du domaine), et pour bien identifier chacun des termes trouvés, il ne sera pas superflu d'en préciser encore le sousdomaine.

Nous n'avons pas jugé indispensable de poursuivre l'identification au-delà du sousdomaine. Nous avons ainsi respecté le principe de simplicité des fiches terminologiques. De plus. dans les pratiques terminologiques actuelles, rares sont les fiches présentant plus de deux niveaux dans la précision du domaine.

Enfin. pour assurer la clarté de la fiche, il a été convenu que le domaine et le sousdomaine y seraient mentionnés en toutes lettres.

\section{FIABILITÉ,}

Les fiches terminologiques sont de valeur inégale. selon leur contenu, d'où la nécessité d'en établir la cote de fiabilité. 
Celle-ci portera sur l'ensemble des informations contenues dans la fiche, mais elle sera principalement déterminée par la moyenne des cotes de fiabilité des différentes sources consultées au moment de la rédaction de la fiche.

La cote de fiabilité des sources a été ainsi établie :

- cote 1: normes:

- cote 2: publications d'associations, de centres de formation, et de spécialistes ;

- cote 3: encyclopédies et dictionnaires monolingues ;

- cote 4: lexiques bilingues ou multilingues :

- cole 5: sources traduites.

À ces cotes s'ajoutent des critères d'évaluation des sources', sur une échelle de 5 points, qui permettent de départager deux ou plusieurs ouvrages du même type.

Ces critères sont les suivants:

- date :

- pertinence des indications bibliographiques:

- présence d'un index :

- réédition ou fort tirage :

- notoriété de l'auteur.

\section{Informatisation de la fiche}

Afin de consigner les données terminologiques recueillies sur un format de fiche uniforme. il fallait choisir un système efficace dans la gestion de ces informations.

Nous visions d'abord une saisie des données efficace. Nous voulions ensuite une présentation claire des fiches, proposant une structure où toutes les informations pertinentes étaient évidentes.

Comme il s'agissait d'un travail de groupe, il fallait un système où chacun puisse idéalement avoir accès à toutes les données à des fins de consultation, mais où seul l'auteur de chaque fiche ait la possibilité de modifier le contenu du document.

Nous avons donc opté pour le système informatisé, qui répond à l'ensemble de ces besoins tout en assurant l'uniformité des fiches.

Aujourd 'hui, il existe quantité de logiciels de base de données sur le marché et même des logiciels conçus pour la gestion des fiches terminologiques, comme Termex. Edibase. Proterm et Multi Termt.

Notre choix s'est porté sur le logiciel de base de données intégré Claris Works, non seulement parce qu'il nous offrait une gestion efficace et une présentation claire des données, mais aussi parce qu'il était très convivial. Parmi ses diverses fonctions, il offre la possibilité de limiter les valeurs possibles pour un champ donné et d'aligner les champs correspondants en anglais et en français à l'écran comme sur papier. Cette dernière fonction est très utile pour mettre en évidence les distinctions entre la partie anglaise, la partie française et la partie administrative de la fiche. 


\section{Exemples de fiches}

\author{
redette.ang crust leather \\ catégorie.eng n \\ remarque.ens uncount \\ defloltion.ang Leather which, after anning, has not \\ been further processed but has \\ merely dried out. (IGLT 1975:20)
}

\author{
vedetle.fr cuir en crouite \\ catcorie.fr $\mathrm{nm}$ \\ remarque.fr non-complable \\ denaltion.tr Cuir qui après avoir été tanne, n'a pas \\ eté autrement travaille, mais simplement \\ seché. (IGLT 1975:87)
}

contexte.fr

En France, le terme s'applique a toutes les sortes ainsi qu'aux parties de cuirs et peaux seulement tannes, exemple mouton en croute, collet en croùte. (TER 05-93)

canned" is similarly employed in

connection with cattic hide leather. (TER 05.93) colloc.fr en croute

renvol.fr crodte, en crodte

gyonyme.tr

obs.ane NOR EN: crust leather. STD-BSI obs.tr

\begin{tabular}{ll} 
domaine & Cuir \\
sous-dom & corroyage \\
flobillte & 3 \\
\hline Initisles & MLH
\end{tabular}




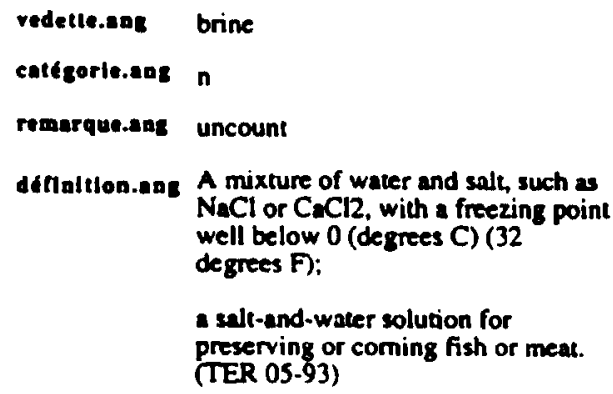

a salt-and-water solution for preserving or coming fish or meat. (TER 05-93)

confexte.ang They are then soaked for $\mathbf{4 8}$ hours in strong brine and drained. after which they are salted. (Wilson 1941:70)

In some countries salt curing is carried out by "Wet salting". whereby the hide is immersed and saturated with brine solution. (UNEP 1991:16)

\section{colloc.ens dip in brine}

renrolung brining

sysoayme.eas

obs.ene

\section{redelte.fr saumure \\ calteorle.fr $\mathrm{nm}$ \\ remarque.fr}

denaition.fr

Eau fortement salke dans lequelle on met des aliments pour en faire des conserves. (TER 05-93) contexte.fr

Les peaux conservées dans la saumure doivent subir un dessalage minutieux. car le sel risque de ntagir avec la chaux (base) de l'opération suivante. (Dupont et Mathieu 1981:115)

colloc.Ir placer une peau dans de la saumure

rearol.fr saumurage

syooayme.fr eau hypersaline

obs.tr

$\begin{array}{ll}\text { domelae } & \text { Cuir } \\ \text { sous-dem } & \text { Conservation } \\ \text { flabilite } & 3 \\ \text { initiales } & \text { MnH } \\ \text { date } & 2003994\end{array}$




vedette.ans cutch
cateforie.ang $n$
remarque.ang uncount
deflaitlon.ane A flavouring extract prepared from
the heartwood of Acacia Catechu ...
used in bitters, fruit and drum
flavourings. (TER 05-93)

contexte.ang In Germany in 1904. Dr. Johannes Paessler demonstrated the superior tanning qualities of mangrove cutch over oak bark, and this started an active interest in the studies which have since led to the discoveries of the remarkable properties of cutch extract. which make it unique among all vegetable tanning materials. (Wilson 1941:282)

colloc.ene

cutch extract

cutch extract

renvol.f

oynonyme.fr caltechu; cachou de Pegu

obs.ens

Use: Tanning, dyeing fabrics brown obs.tr and black.

vedettefr cachou
categorle.fr $\mathrm{nm}$
remarque.fr

dennition.fr Substance noire, brillante, cassante. soluble dans l'esu boulllante, extraite de la noix de betel (fnuit d'Acacia catechu). Elle est utiliste pour obeenir le pyrocathocol et ses dérives et notamment pour ses propriétés astringentes. tannantes et antidiarticiques. (TER OS. 93)

contexte.fr

Le constituant principal du cachou officinal ou cachou de pegu est le tanin. accompagné de caltechine et d'acide cachoutannique. Il sert a préparer la teinture de cachou. (TER 05-93)

\begin{tabular}{|c|c|}
\hline domaine & Cuir \\
\hline sous-dom & Tannage \\
\hline neblilte & 4 \\
\hline Initiales & MIH \\
\hline date & $20103 / 94$ \\
\hline
\end{tabular}




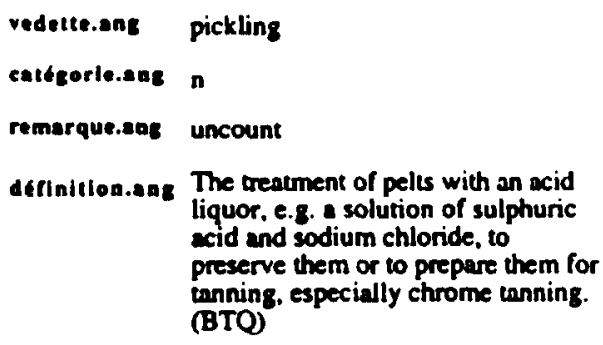
liquor. e.g. solution of sulphuric acid and sodium chloride, to preserve them or to prepare them for innning, especially chrome tonning. (BTQ)

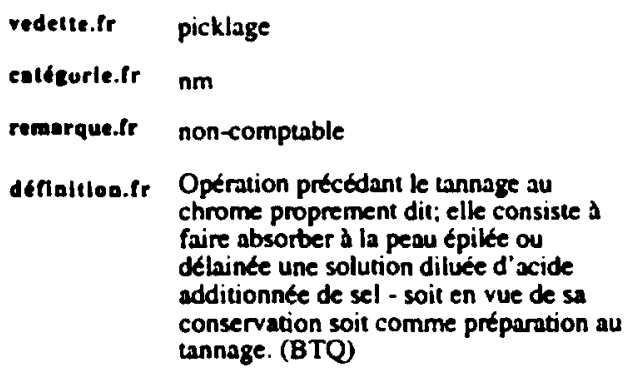
chrome proprement dit; elle consiste a faire absorber a la peou épike ou délainte une solution diluee d'acide additionné de sel - soit en vue de sa conservation soit comme preparntion au tannage. (BTQ)

contexte.fr Laisser tremper une peau dans une
solution d'acide avant de la frotter avec
de la cervelle (reproduction en
laboratoire d'une technique
amérindienne) ne reprend que la phase
de picklage par laquelie certains tanneurs
terminent le dechnulage et preparent les
peaux au tannage proprement dit.
(Dupont et Mathieu $1981: 116$ )

colloc.fr

reavol.fr

obs.fr

demalae

sone-dom

nablite

Ialtiales

date

\section{Cuir}

Industrie du cuir, Maroquinerie

MIH

0910494 


\title{
CONCIUSION
}

Le présent travail terminologique a permis de confirmer que le cuir. plus particulièrement sa fabrication, était un très bon exemple par rapport à l'objectif que nous nous étions fixé. Nous avons pu constater que la méthodologie utilisée est efficace et qu'elle s'avère être applicable à tous les domaines. En effet, les termes utilisés sont empruntés pour la plupar à la langue courante (par exemple. les termes de cuisine comme «nourriture») et les collocations foisonnent dans ce domaine (entre autres, on dira abaisser les têtes» pour amincir la partie épaisse du cou et de la tête des bovins et "abreuver une fosse" pour recouvrir d'eau ou de jus tannant les cuirs qui sont empilés entre les couches de tan).

Nous espérons que notre travail et les conclusions qui en découlent permettront à d'autres terminologues de mener à bien ce genre d'étude è qu'il donnera envie à certains de poursuivre cetlé recherche, le travail de défrichement ayant été fait.

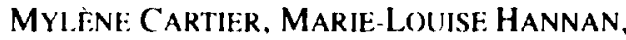
NADIA MOUILAY ET JEAN NTAKIRUTIMANA

\author{
ANNEXE: 1 : REFERENCES HIBLIOCRAPHIQUES SUR LFCUIR \\ Sources primaires \\ NORMFS

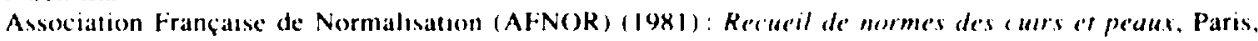 \\ AFNOR, 247 p. \\ ISO $282(1(1474)$ : ( 'uir Pealux hrutes de bovides et d'eyundes \\ ISO $2821(1974)$ : ('urr Peaux hrutes de bovides et dequidés \\ Mode de presentalion. \\ Comservattion par salage en pile
}

OFFICI: DIES NORMES DU GOUVERNIMENT ( ANADIEN

CGSB : 5-(;P-2.3 leather : caltlehide, vegetable tanned, case

CGSB : 5-(iP-36 leather: cattlehide, upholstery.

(ciSB:5-(ip-1.3 Peaux de chamons. (Angl-fr.)

ONC;C : 5 -6ip-22 Cuir pour garnitures hydrauliques

MONOCIRAPHIES YTI PUBLIC 'ATIONS OFFIC IEILI.ES

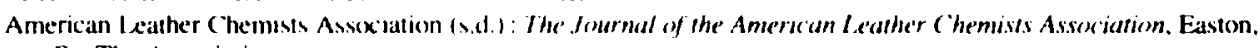
Pa. The Association.

CHURCHIl.l., J. F., (198.3): The ('omplefe Besok of Tamning Skins and Furs, Harrishurg. Pa. Stackpole Books. $197 \mathrm{p}$.

Collecuf (1975): Timner, leindre. fims, Leverkusen, Bayer. 2.54 p.

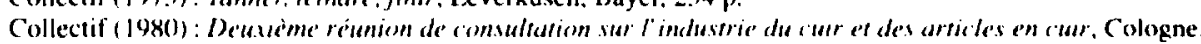

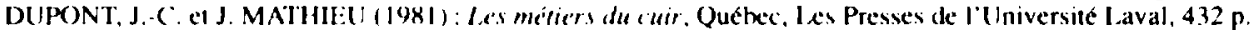

Fond and Agriculture (Organmation of the I Inited Natons ( I 961$)$ : Rural Tamning Téchniques, FAO). 251 p.

GARANT, Y. a d (IU78): Le' 'ur. Montreal, Publications Mandarine, Collection des Artisans québecois, 94 p.

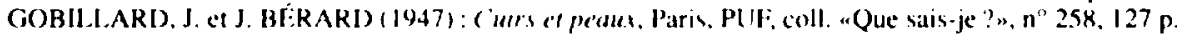

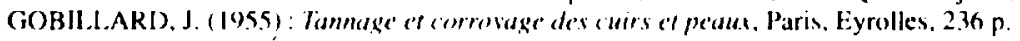

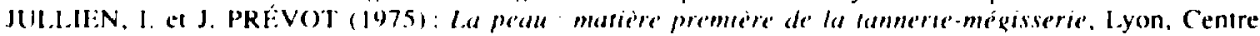
technique du cuir, $44 \mathrm{p}$.

KEDDIE. J. (1981): Tanning of Hales amd Skans, (iveneva, International lahour ( )ffice.

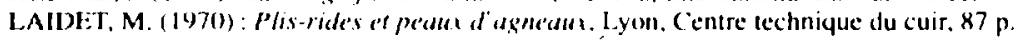

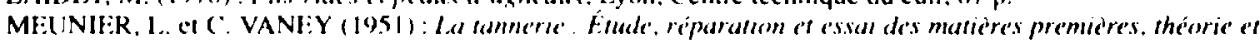

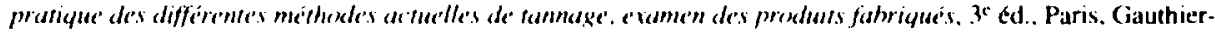
Villars, 2 t. $918 \mathrm{p}$. ct $58.3 \mathrm{p}$.

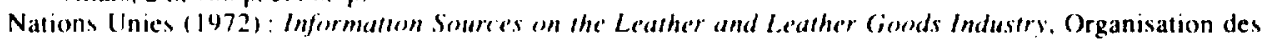
Nations (Inies pour le Ieveloppement Industriel, $80 \mathrm{p}$.

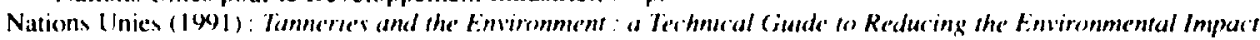
of Tommery Operatiens, Ist Ed., Paris, U.N. Environment Program. Industry and Environment Office. Technical rejurt series $n^{\prime \prime} 4$.

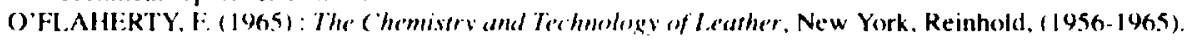


Organisation des Nations Unies pour l'alimentation et l'agriculture. Division de la mise en valeur des terres et des eaux. Sous-division du génie rural (1962): Méthodes artisanales de pannage. Rome. FAO, XVIII. réimpr. 1978. $256 \mathrm{p}$.

PORÉ. J. (1974) : La nourriture du cur : Méthodes el principes, Paris. Sociéle des publications "Le cuir». VIII, $362 \mathrm{p}$

RAY. L. R. (1951) : Equipment for Tanning of Hides and Skins, FAO.

ROGERS, A. (s.d.): Methodes modernes de fabrication des cuirs es peaux, Paris, Gauthier-Villars, XIII, $608 \mathrm{p}$. THORSTENSEN. T. C. (1969) : Practical Leather Technology, New York. Van Nostrand Rienhold Co., 272 p.

WILSON, J. A. (1941) : Medern Practice in Leather Manufacture. New York, Reinhold, 744 p

Sources secondaires

DICTIONNAIRES, GLOSSAIRES ET LEXIQUES

Centre technique du cuir (1972): Thésourus cuir et chaussure, Lyon. Centre technique du cuir.

Conseil intemational des tanneurs (1975): International Glossary of Leather Terms. 2nd Ed.. Londres, International Council of Tanners. $320 \mathrm{p}$.

Dichonnaire du cuir anglais, français. allemand, ilalien. russe. espagnol (1976): Paris, La Maison du dictionnaire. $720 \mathrm{p}$

Ecole française de tannerie, Lexique de tannerie, $10 \mathrm{p}$

GATT (1970) : Glossaire du cuir ò l'usage des traducteurs du (GATT. Geneve. GATT. $71 \mathrm{p}$

Union Internationale des societes des lechniques et des chimistes des industries du cuir (1976): Leather Terhnical Gilessary in Sin Languages, Darmstad (RFA)

\section{ENCYCLOPÉDIES}

American Heritage Dictionary (on Microsoft Bookshelf 1992 on CD-ROM).

The Canadian Enc yclopedia (1988): Edmonton. Hurtig Publishers.

Collier's Concise Enc velepactia (on Microsoft Bookshelf 1992 on CD-ROM)

Encvelopadia Britannic a (199) ): London, Encyclopadia Britannica Inc.

Encyclopadia liniversalis (1984): Paris, Encyclopaedia Universalis.

(irand Larousse de la langue franciaise (1978): Paris, Larousse.

Cirand Larousse Enc vclopédique en 10 volumes (1982): Paris, Larousse.

Larousse if velume's en irouleurs (1966): Paris, Larousse.

The World Bose Encyclapedia (1993): Toronto. Field Enterprises Educational Corporation.

\section{ANNEXE 2 : LEXIQUE DU CUIR}

abattoir

acid burn

acid burn caused by urine

adipose layer

adipose tissue

agglutination

alkaline solution

ammonium chloride

ammonium sulfate

angle of weave

aniline dyes

appendage

area measured leather

area leather

areolar tissue

anificial grain (pattern)

assisted moroceo

automotive leather

back

backbone

bag leather

barbed wire scratch abattoir

brùlure par acide

brülure par acide causée par l'urine

couche adipeuse

IIssu adipeux

graisse

agglutination

solution alcaline

chlorure d ammonium

sulfate d ammonium

angle d'entrelacement

couleur d'aniline

couleur a l'aniline

colorant d'aniline

appendice

surface du cuir

surface du cuir

tissu arélaire

IIssu

neur artificielle

cheyre grain maroquin

cuir pour rembourrage auto

dosset

raie du dos d'une peau

cuir pour sacs

eratlure de fil de fer 


\begin{tabular}{|c|c|}
\hline $\begin{array}{l}\text { basil } \\
\text { bating material }\end{array}$ & $\begin{array}{l}\text { basane } \\
\text { confits }\end{array}$ \\
\hline bating & $\begin{array}{l}\text { conftis } \\
\text { confitage }\end{array}$ \\
\hline beaver lamb & agneau-castor \\
\hline bellows hide & cuir à soufflet \\
\hline belly & flanc \\
\hline helly split & croûte de flanc \\
\hline belly grain & flanc scié \\
\hline belly middle & milieu de flanc \\
\hline belt split & croûte à ceinture \\
\hline $\begin{array}{l}\text { belt leather } \\
\text { belting butt }\end{array}$ & $\begin{array}{l}\text { cuir a ceinture } \\
\text { croupon a courrole }\end{array}$ \\
\hline bend range & $\begin{array}{l}\text { croupon à courrole } \\
\text { bande de croupon }\end{array}$ \\
\hline bend & croupmon \\
\hline bichromate of scoda & bichromate de soxlium \\
\hline $\begin{array}{l}\text { binder leather } \\
\text { bleach }(n)\end{array}$ & $\begin{array}{l}\text { cuis pour frein a friction } \\
\text { agent de blanchiment }\end{array}$ \\
\hline bleach (to) & blanchur \\
\hline blister & $\begin{array}{l}\text { phlyctenc } \\
\text { hoursouflure }\end{array}$ \\
\hline bloxd albumen & albumine sanguin \\
\hline bloxd lipid & lipides sanguins \\
\hline hlood serum & serum sanguin \\
\hline borkbinding leather & cuir de reliure \\
\hline herot nappa & hotte nappa \\
\hline bottom leather & cuir a semelage (a semelle) \\
\hline bovine calf & veau \\
\hline $\begin{array}{l}\text { hovine wet blues } \\
\text { bovine hide }\end{array}$ & $\begin{array}{l}\text { curr de bovin en bleu humide } \\
\text { peaux de bovins }\end{array}$ \\
\hline box side leather & $\begin{array}{l}\text { hatnde valchelle-box } \\
\text { vachette-trox }\end{array}$ \\
\hline box side & $\begin{array}{l}\text { bande vachetle-box } \\
\text { vachelle-box }\end{array}$ \\
\hline box calf & (x) $x$-cialf \\
\hline brand mark & marque figurative \\
\hline break & bre:il \\
\hline grain break & clatement de lat fleur \\
\hline breast edge & montant \\
\hline breast collar & $\begin{array}{l}\text { bricole } \\
\text { pesitrinière } \\
\text { collier }\end{array}$ \\
\hline bridle leather & cuir à bride \\
\hline brine cured & saumuré \\
\hline brine curing & saumurage \\
\hline brine & $\begin{array}{l}\text { saumure } \\
\text { cau hypersialine }\end{array}$ \\
\hline $\begin{array}{l}\text { brine-cured limed split } \\
\text { brining }\end{array}$ & croûte saumurée-chauléc \\
\hline brushed pigsk in & $\begin{array}{l}\text { peiau de pore velours } \\
\text { pore velours }\end{array}$ \\
\hline pigskin suede & $\begin{array}{l}\text { peial de pore velours } \\
\text { porc velours }\end{array}$ \\
\hline buckskin & daim \\
\hline huff & chamoss \\
\hline buff culf & buff cilf \\
\hline buffalo leather & buffle \\
\hline buffed leather & $\begin{array}{l}\text { cuir a fleur corriges } \\
\text { cuir a fleur tanllé }\end{array}$ \\
\hline bulge & boursouflure \\
\hline bull split & titureau croûte \\
\hline bull hide & perau de taureat \\
\hline bundle & mettre en balle \\
\hline
\end{tabular}




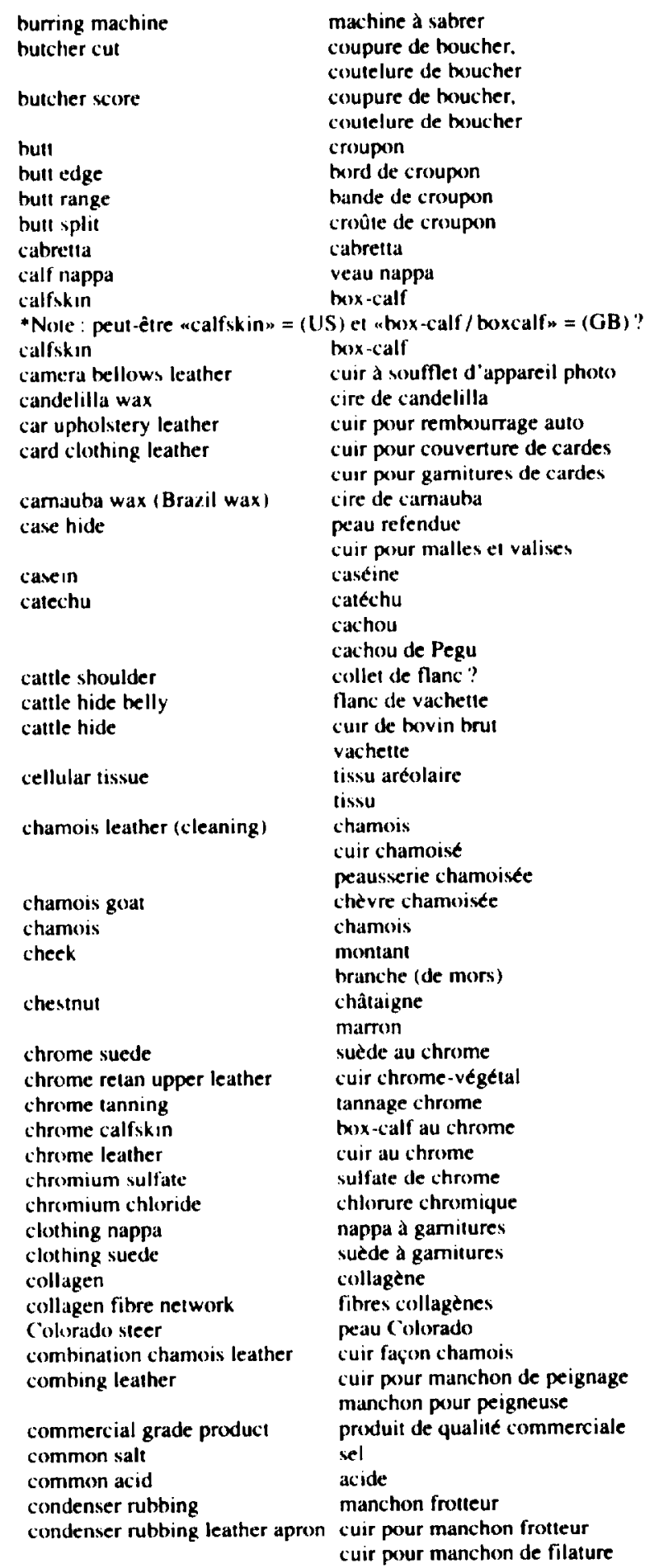




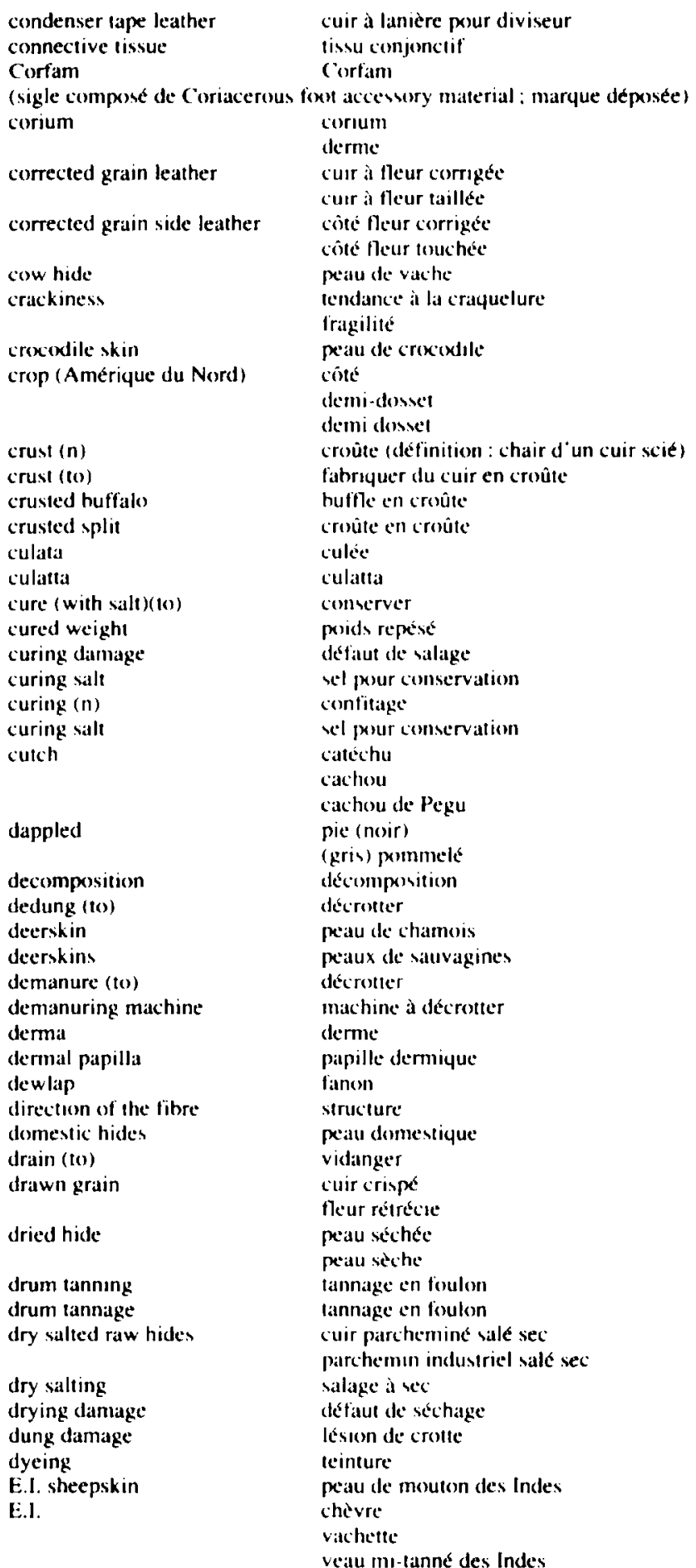




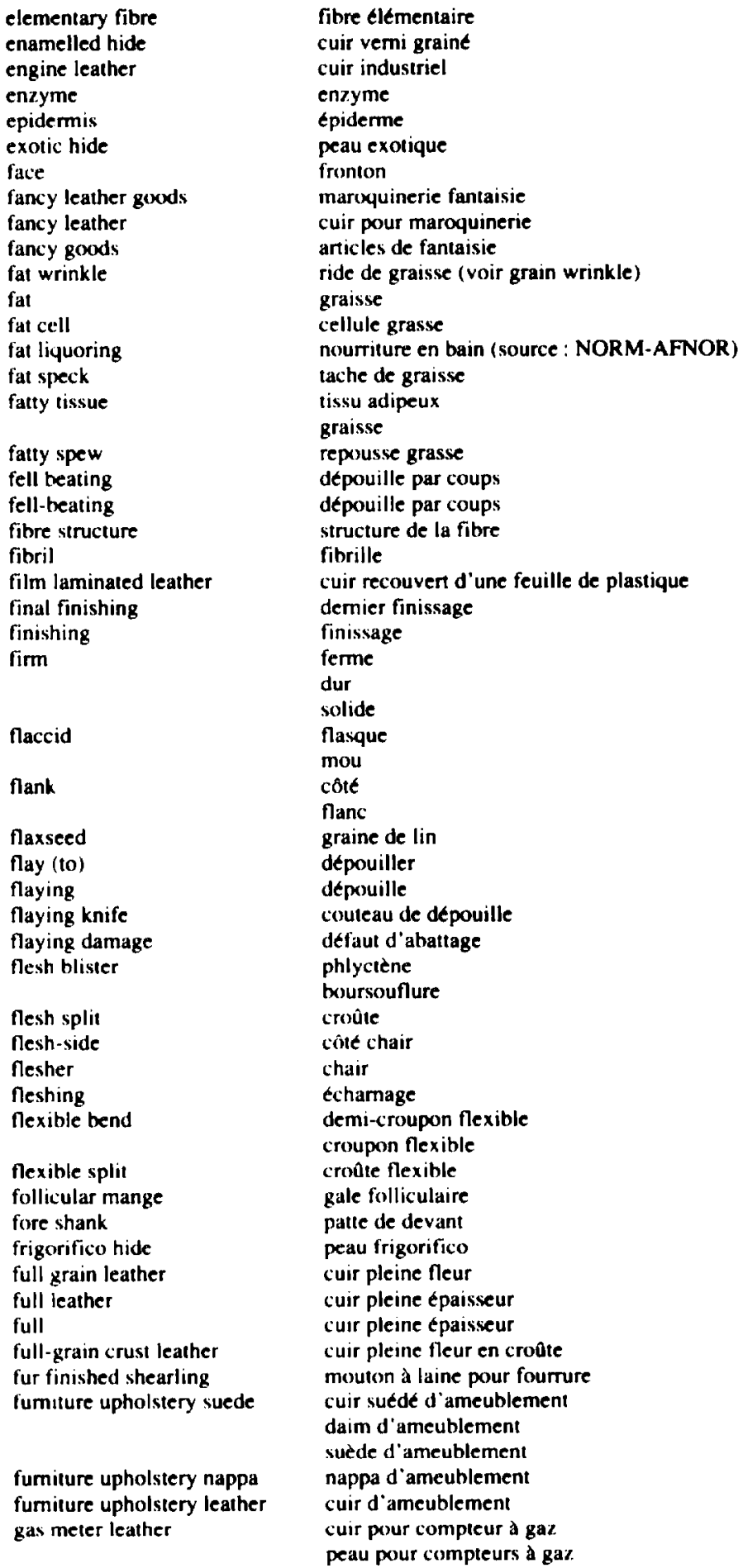




\begin{tabular}{|c|c|}
\hline gash & $\begin{array}{l}\text { déchirure } \\
\text { entaille }\end{array}$ \\
\hline gear leather & cuir a hamachement \\
\hline $\begin{array}{l}\text { genuine Russia calf } \\
\text { glace goat }\end{array}$ & $\begin{array}{l}\text { (cuir de) veau de Russie véritable } \\
\text { chère glacé }\end{array}$ \\
\hline glace kid & chevreas glaces \\
\hline glaze(to) & lustrer \\
\hline glazed kid & chevreau glaces \\
\hline glohulin & globuline \\
\hline gloving leather & curr pour ganterie \\
\hline gloving kid & $\begin{array}{l}\text { chevreatu ganterie } \\
\text { chevreatu pour ganterie }\end{array}$ \\
\hline glue-stcxk & carnasses \\
\hline goad mark & $\begin{array}{l}\text { marque d'aiguillon } \\
\text { piqûre d'aliguillon }\end{array}$ \\
\hline goatskin & chevere \\
\hline goatskin patent leather & peatu de caprins \\
\hline $\begin{array}{l}\text { goatskin patent leather } \\
\text { goatskin gloving leather }\end{array}$ & $\begin{array}{l}\text { cuir verni en peau de caprins } \\
\text { cuir pour ganteric en peau de caprins }\end{array}$ \\
\hline goatskins & peaux de caprins \\
\hline goatskiver & scee de chevre \\
\hline good-quality hide & peau de bonné qualıté \\
\hline gouge & balsse \\
\hline grain & fleur \\
\hline grain wrinkle & ride de tleur \\
\hline grain leather & cuir fleur \\
\hline grain layer & $\begin{array}{l}\text { peau lravantec cote fleur } \\
\text { fleur ou couche superficielle } \\
\text { cote poil }\end{array}$ \\
\hline grain pattern & dessin de la fleur \\
\hline grain structure & structure de fleur \\
\hline grain split & Me'ur refienduc \\
\hline grain pattern & fletur \\
\hline & dessin de la fleur \\
\hline grain side & $\begin{array}{l}\text { côté fleur } \\
\text { fleur } \\
\text { côté poil } \\
\text { poil } \\
\text { facce externe }\end{array}$ \\
\hline grain defect & défaut de fleur \\
\hline graining & légeage \\
\hline green weight & poids vert \\
\hline green hide & $\begin{array}{l}\text { peau fraiche } \\
\text { peadu vertic }\end{array}$ \\
\hline green salt. (to) & viler vert \\
\hline green-salted & sale frais \\
\hline grun boil & trou de varron \\
\hline gum tragacanth & $\begin{array}{l}\text { goumme adragante } \\
\text { adragant(e) } \\
\text { adraganthe }\end{array}$ \\
\hline hair sheep & mouton a poils \\
\hline hair-slip (USA) & $\begin{array}{l}\text { peau echauflec } \\
\text { echauffe } \\
\text { echautfurs }\end{array}$ \\
\hline hair & punl \\
\hline hair papilla & papille du poil \\
\hline hair slip & $\begin{array}{l}\text { peau echauffec } \\
\text { echauffure }\end{array}$ \\
\hline hairsheep & $\begin{array}{l}\text { schautie } \\
\text { mouton poilu }\end{array}$ \\
\hline halfback (v. crop) & côte \\
\hline & $\begin{array}{l}\text { demi-dosset } \\
\text { demi dosset }\end{array}$ \\
\hline
\end{tabular}




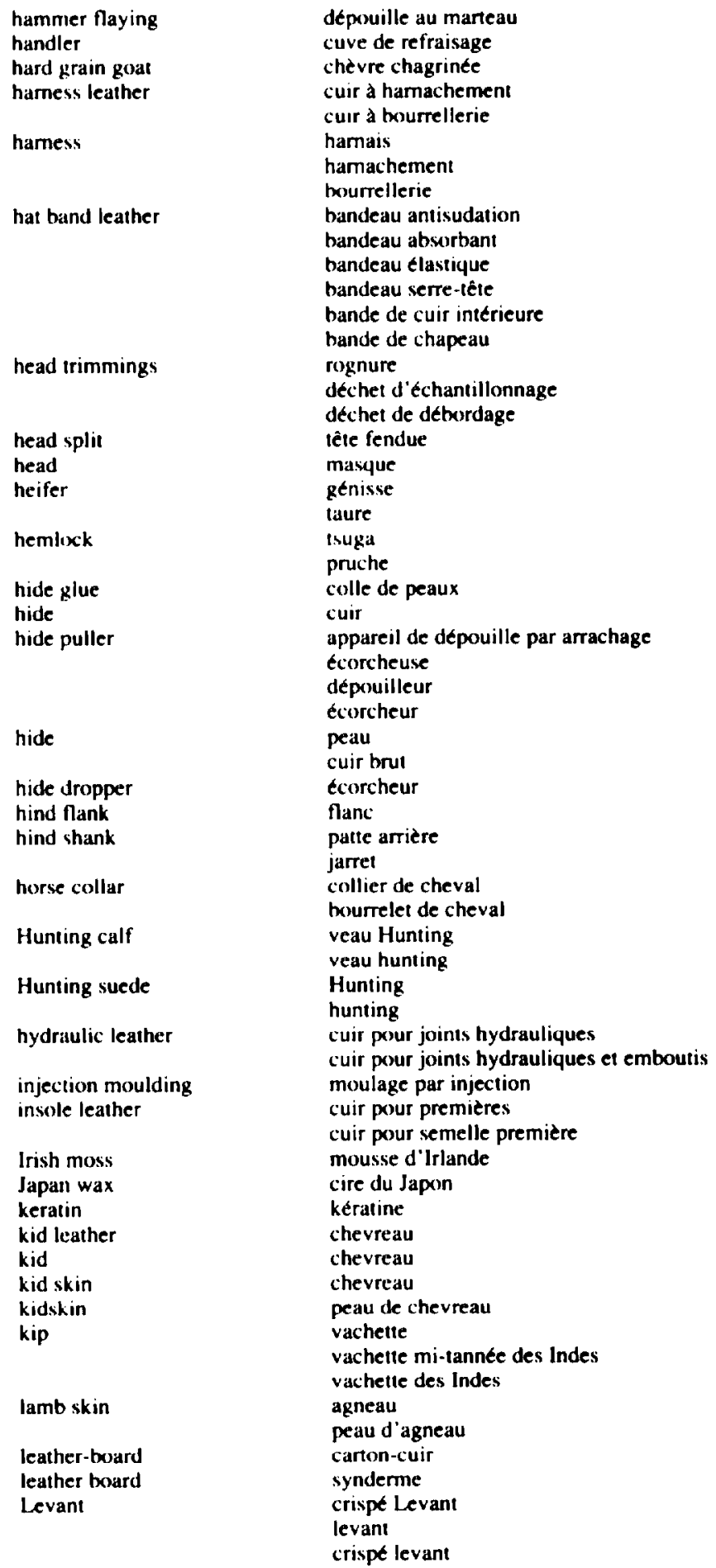




\begin{tabular}{|c|c|}
\hline levelled bend & $\begin{array}{l}\text { croupon egalive: } \\
\text { demi croupon egalise } \\
\text { croupon egalive }\end{array}$ \\
\hline lifting leather & cusr pour sous bxuts \\
\hline $\operatorname{lime}$ & chatux \\
\hline lining leather & $\begin{array}{l}\text { perau à doublure } \\
\text { couir à doublure } \\
\text { coirs à doublures }\end{array}$ \\
\hline lining shearling & p'au a doublure nalurelle \\
\hline liquor & liqueur \\
\hline machine buff & $\begin{array}{l}\text { ponçage machine } \\
\text { curr poncé }\end{array}$ \\
\hline mangrove & mangrove \\
\hline manufacturing bend & demi-croupon pour la fabrication de chaussures \\
\hline mechanical flayer & conteinu ì dépouiller mécanique \\
\hline melallized leather & cuir metallisés \\
\hline mid-sole leather & cuir i semelle intercalaire \\
\hline milling ( $n$ ) & foulage \\
\hline mineral tanning & Lannage mineral \\
\hline $\begin{array}{l}\text { mixed tannage bottom leather } \\
\text { mixed (vegetable) tannage }\end{array}$ & $\begin{array}{l}\text { suir à semelage obtenu par tannage végétal mixte } \\
\text { tammage végétal mixte }\end{array}$ \\
\hline morecos grained goat & chevre grain marequin \\
\hline mould control & controle de conformite \\
\hline mould soles (10) & emboutir les semelles \\
\hline mould stain & lache de moisissure \\
\hline mouldy & moist \\
\hline mucous layer & $\begin{array}{l}\text { southe de Malpighi } \\
\text { sorps muqueux de Malpighi }\end{array}$ \\
\hline mulling ( $n$ ) & humidilicalion \\
\hline nap & ironton \\
\hline nappa & nappa \\
\hline native hides & peau domestique \\
\hline natural grain & grain naturel \\
\hline natural & naturel \\
\hline natural appearance & aspect naturel \\
\hline natural fecl & lowcher naturel \\
\hline natural thickness & pleine épaisseur \\
\hline nubuck & nubuck \\
\hline oak & chêne \\
\hline offal & dépouille \\
\hline One-Bath Method (proxess) & procédé en bain unique \\
\hline padctle val & coudreuse (à confit) \\
\hline orthopaedic leather & cuir orthopédique \\
\hline oxe hide & peau de bxuf \\
\hline packer hide & péau de packer \\
\hline packing leather & cuir pout joints hydrauliques et emboutis \\
\hline papilliary layer (skin) & couche papillatire \\
\hline parchmnent & parchesmin \\
\hline pasting & sollage \\
\hline & (collage des cuirs sur cadre) \\
\hline patent leather (laminated) & cuir verm plaqué \\
\hline patent split & croûte de cuir vermic \\
\hline patent leather & cuir verni \\
\hline pearlized leather & suir nakré \\
\hline peccary & peccarı \\
\hline peg out $(t))$ & surer à l'aude de chevilles \\
\hline pelt & p'au con tripe \\
\hline Persian & metis des Indes \\
\hline picking band leather & cuir pour fonet de chasse \\
\hline pickle (10) & pickler \\
\hline pickled goatskin & peau de chevre pioklese \\
\hline
\end{tabular}




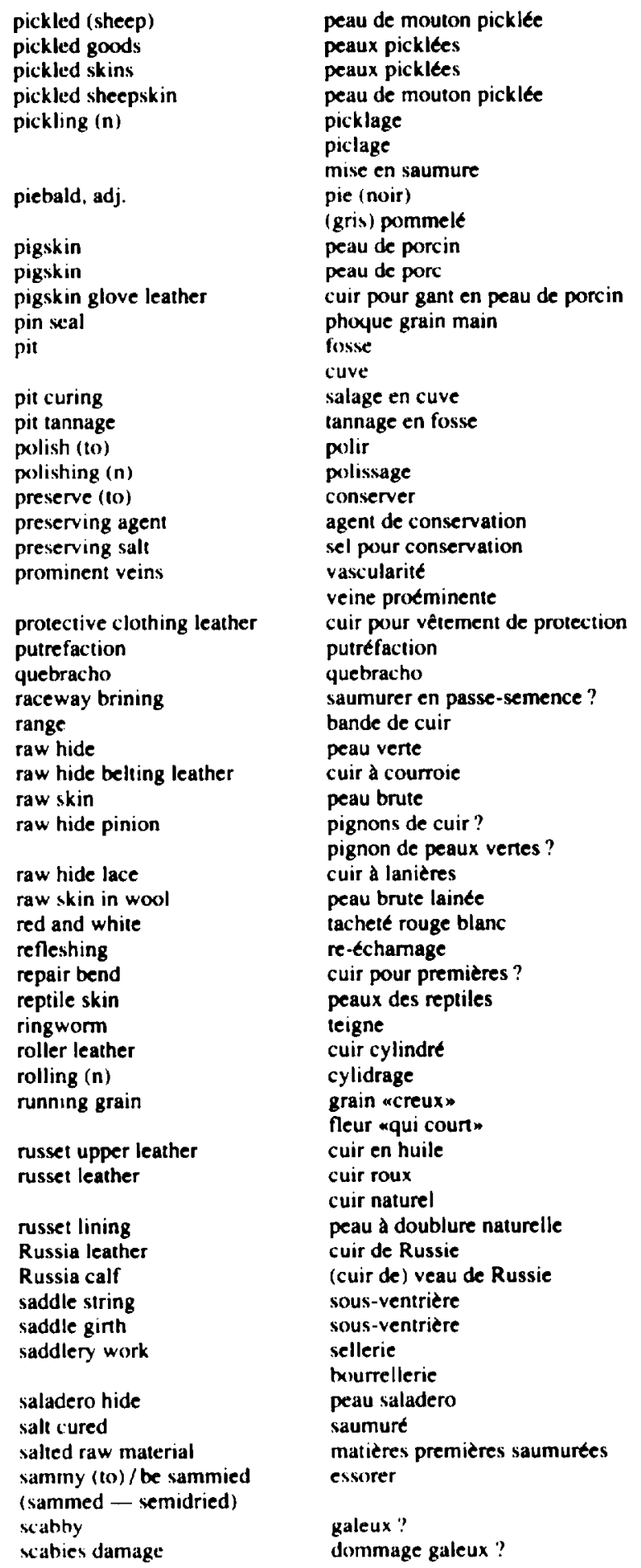




\begin{tabular}{|c|c|}
\hline scalding damage & taches d'echaudage? \\
\hline scored & corutele \\
\hline scratch & $\begin{array}{l}\text { Egratant } \\
\text { griffes }\end{array}$ \\
\hline scud $(t o)$ & $\begin{array}{l}\text { décrasier } \\
\text { façonner }\end{array}$ \\
\hline scud & cratsse \\
\hline scudding (n) & decrassage \\
\hline sebacious gland & glande sebacese? \\
\hline sectioning of the hide & secetulu de peau \\
\hline semi-finished skins & cuir sem-fini \\
\hline set & déforme de lisse \\
\hline shagreen & chagrm \\
\hline shank & $\begin{array}{l}\text { cambrion } \\
\text { cambrillon }\end{array}$ \\
\hline shank trimmings & $\begin{array}{l}\text { coupcaux } \\
\text { rognures de cambrion } \\
\text { cambrillon }\end{array}$ \\
\hline sheep pel & tpe:au de moutons \\
\hline sheep upper leather & cuir de mouton a dessus? \\
\hline sheepskin & peatu cle mouton (tannee) \\
\hline $\begin{array}{l}\text { sheepskin clothing leather } \\
\text { sheepskin }\end{array}$ & $\begin{array}{l}\text { peau de mouton pour vêtements } \\
\text { mouton retourné }\end{array}$ \\
\hline sheepskin (with weol) & $\begin{array}{l}\text { peilu de mouton } \\
\text { peatu de mouton lainée. } \\
\text { peiald de mouton en laine }\end{array}$ \\
\hline shell & cuir culec \\
\hline shellac & $\begin{array}{l}\text { lacque en ecalles } \\
\text { vernis a la gomme-lacque }\end{array}$ \\
\hline shoe nappa & nappa pour chaussures \\
\hline shore suede & suede de chatussures \\
\hline shoe split & cuir fendu pour empeignes \\
\hline shoe lining leather & cuar pour doublures de chaussures \\
\hline shoc upper split & cuir à dessus pour chaussures \\
\hline $\begin{array}{l}\text { shor-length wor)l sheepskin } \\
\text { shoulder }\end{array}$ & $\begin{array}{l}\text { peitu de moutom à lante counc } \\
\text { collet }\end{array}$ \\
\hline shoulder grain & collet scis \\
\hline side upper leather & cuir de collet à dessus? \\
\hline side & tlanc \\
\hline side clothing leather & cuir de flanc pour les vêtements \\
\hline side nappa & nappa de llanc \\
\hline side nubuck & mubuck de flanc \\
\hline side shoulder & collet de flanc? \\
\hline side leather & cuir de flanc \\
\hline silky sheen suede & velours changeant \\
\hline vilver leather & peill argent \\
\hline skin & peilu \\
\hline skin lacking substance & absence de coloration de peau \\
\hline skin fat & matiere grasse \\
\hline skin collagen & collagene \\
\hline skirting leather & cuir pour panncaux de selle \\
\hline skiver & peall de monson refendue \\
\hline slat & cuirot \\
\hline slatted weight & poids repente \\
\hline slaughterhouse & abattour \\
\hline slink lamb & mort-ni"? \\
\hline $\operatorname{smovoth}(t 0)$ & lisucer \\
\hline smoxthing (n) & linsilge \\
\hline soaking & trempe \\
\hline & reverdissage \\
\hline sodium sulfide & sulfure de sodium \\
\hline sodium thiosulfatethio & sulfate de sodium \\
\hline
\end{tabular}




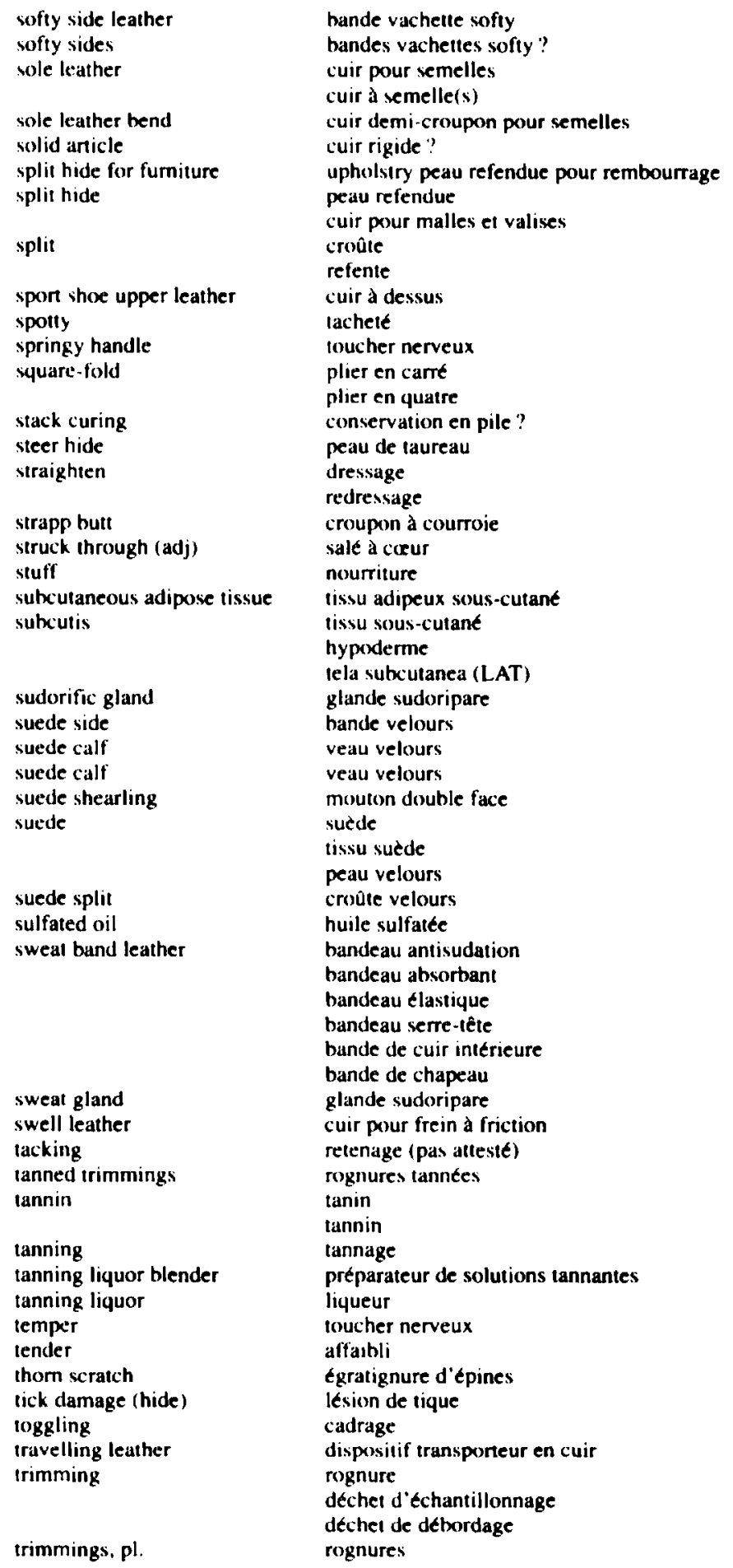




\begin{tabular}{|c|c|}
\hline two-coloured (hide) & Ple (noir) \\
\hline two-way suede & $\begin{array}{l}\text { (gris) pommele } \\
\text { velours changeiant }\end{array}$ \\
\hline Two-Bath Methexd & procedle en deux bains \\
\hline undercoat of hair & $\begin{array}{l}\text { twourre } \\
\text { duvet }\end{array}$ \\
\hline underfur & $\begin{array}{l}\text { mourre } \\
\text { duvel }\end{array}$ \\
\hline unhairing shop & atelerer d'épilinge \\
\hline unhairing & $\begin{array}{l}\text { depilation } \\
\text { ejarrage } \\
\text { epilage" }\end{array}$ \\
\hline untanned trimmings & $\begin{array}{l}\text { epilage" } \\
\text { rognures non tannees }\end{array}$ \\
\hline $\begin{array}{l}\text { unweaned lamb } \\
\text { upholstery leather }\end{array}$ & $\begin{array}{l}\text { agneau blane } \\
\text { curr d'aneublement } \\
\text { curr pour rembourrage }\end{array}$ \\
\hline upper leather & $\begin{array}{l}\text { curr pour de'ssus de chaussures } \\
\text { cuir il dessus }\end{array}$ \\
\hline $\begin{array}{l}\text { upperleuther split } \\
\text { varnish }\end{array}$ & $\begin{array}{l}\text { cuirs fendus pour empergenes } \\
\text { vernis }\end{array}$ \\
\hline vascularity & $\begin{array}{l}\text { vascularite } \\
\text { veane prosemunente }\end{array}$ \\
\hline vat & cuve \\
\hline val curing & sallige en cuve \\
\hline $\begin{array}{l}\text { veal skin } \\
\text { veial boix }\end{array}$ & peitu de veau de lait moyen \\
\hline $\begin{array}{l}\text { veal bax } \\
\text { vealer }\end{array}$ & $\begin{array}{l}\text { box-calf } \\
\text { veatu de busucherie }\end{array}$ \\
\hline & $\begin{array}{l}\text { veau de lait } \\
\text { veau clair } \\
\text { veatu rose }\end{array}$ \\
\hline vegetable tanned crust & croûtc tanne végétal \\
\hline $\begin{array}{l}\text { vegetable tanning } \\
\text { vegetable tanned leather }\end{array}$ & lannage végetial \\
\hline $\begin{array}{l}\text { veininess }\end{array}$ & $\begin{array}{l}\text { curr tanne vegetal } \\
\text { vascularile } \\
\text { veine prokeminente }\end{array}$ \\
\hline velvet leather & peatu velours \\
\hline warble grub hxil & trou de vitrron \\
\hline warble boil & trou de varron \\
\hline warble hole & trou de varron \\
\hline $\begin{array}{l}\text { warble fly } \\
\text { wash leather }\end{array}$ & $\begin{array}{l}\text { hypodernce } \\
\text { chamois }\end{array}$ \\
\hline & $\begin{array}{l}\text { chamois } \\
\text { cuir chamoise }\end{array}$ \\
\hline $\begin{array}{l}\text { waterproof leather } \\
\text { wattle }\end{array}$ & $\begin{array}{l}\text { péausserie chamoisée } \\
\text { cuir imperméable }\end{array}$ \\
\hline waltle & $\begin{array}{l}\text { acacia } \\
\text { mimosal }\end{array}$ \\
\hline weight class & classe de poids \\
\hline welting leather & cuir pour trépontes \\
\hline welting belly & flanc a trepointe \\
\hline wet-salt. (to) & sileer vert \\
\hline wet blues & $\begin{array}{l}\text { bleu humicle } \\
\text { cuir en bleu humide }\end{array}$ \\
\hline white hide leather & cuir blanc pour lacets \\
\hline $\begin{array}{l}\text { white lace leather } \\
\text { willow side }\end{array}$ & cuir blanc pour lacets \\
\hline & $\begin{array}{l}\text { bande vachette-hox } \\
\text { vachelle-hox }\end{array}$ \\
\hline willow side & $\begin{array}{l}\text { vachetle } \\
\text { boxcult colors }\end{array}$ \\
\hline willow calf & $\begin{array}{l}\text { boxcailf colors } \\
\text { boxcialf coloré }\end{array}$ \\
\hline uring machine & machine a siabrer \\
\hline wool(l)ed skin & pe:aut laince \\
\hline
\end{tabular}




$\begin{array}{ll}\text { wooled sheepskin } & \begin{array}{l}\text { mouton lainé } \\ \text { mouton en laine } \\ \text { ride }\end{array} \\ \text { wrinkle (hide) } & \end{array}$

Notes

1. Laccessibilité à la documentation ne pourrait étre considérée un critère en tant que tel. Cependant, avec les moyens informatiques dont nous disposons de nos jours, il est possible de recenser une documentation abondante. Il faut s'assurer alors que cette demière est accessible.

2. Selon de Bessé (Meta 36-1 : 112-113). le contexte definitoire est surtout utilisé pour pallier la carence d'éléments de definition.

3. Brunette, 1. (1992): Vocahulare anglais-français du clavecin. Montréal. Presses Unıversitaires de Montréal.

4. La date, les initiales, la fiabilite, le domaine et le sous-domaine appartiennent a la calégorie des rubriques administratives.

5. (Voir note $n^{\circ}$ 3.)

6. Voir Maurice. E. et al. (1991): “Comparaison de trois logiciels utilisables en terminologie: FOXBASE+, MC4, TEXTO», Meia, 36-1. (mars 1991). pp. 207-213. 